\title{
Consistent 4D Cortical Thickness Measurement for Longitudinal Neuroimaging Study
}

\author{
Yang $\mathrm{Li}^{1}$, Yaping Wang ${ }^{2,1}$, Zhong Xue ${ }^{3}$, Feng Shi ${ }^{1}$, Weili Lin ${ }^{1}$, \\ Dinggang Shen ${ }^{1}$, and The Alzheimer's Disease Neuroimaging Initiative* \\ ${ }^{1}$ Department of Radiology and BRIC, \\ University of North Carolina at Chapel Hill, USA \\ ${ }^{2}$ Department of Automation, Northwestern Polytechnical University, Xi'an, \\ Shaanxi Province, P.R. China \\ ${ }^{3}$ Methodist Center for Biotechnology and Informatics, \\ The Methodist Hospital Research Institute, Weill Cornell Medical College, \\ and Department of Radiology, The Methodist Hospital, USA
}

\begin{abstract}
Accurate and reliable method for measuring the thickness of human cerebral cortex provides powerful tool for diagnosing and studying of a variety of neuro-degenerative and psychiatric disorders. In these studies, capturing the subtle longitudinal changes of cortical thickness during pathological or physiological development is of great importance. For this purpose, in this paper, we propose a $4 \mathrm{D}$ cortical thickness measuring method. Different from the existing temporal-independent methods, our method fully utilizes the $4 \mathrm{D}$ information given by temporal serial images. Therefore, it is much more resistant to noises from the imaging and pre-processing steps. The experiments on longitudinal image datasets from the Alzheimer's Disease Neuroimaging Initiative (ADNI) show that our method significantly improves the longitudinal stability, i.e. temporal consistency, in cortical thickness measurement, which is crucial for longitudinal study. Power analysis of the correlation between cortical thickness and Mini-Mental-Status-Examination (MMSE) score demonstrated that our method generates statistically more significant results when comparing with the $3 \mathrm{D}$ temporal-independent thickness measuring methods.
\end{abstract}

\section{Introduction}

Many recent anatomical magnetic resonance image (MRI) studies on the human brain have been focused on the cerebral cortex thickness analysis, because longitudinal variations in cortical thickness are found closely correlated to either

\footnotetext{
* Data used in the preparation of this article were obtained from the Alzheimers Disease Neuroimaging Initiative (ADNI) database (www.loni.ucla.edu/ADNI). As such, the investigators within the ADNI contributed to the design and implementation of ADNI and/or provided data but did not participate in analysis or writing of this report. ADNI investigators include (complete listing available at www.loni.ucla.edu/ADNI/Collaboration/ADNI_Authorship_list.pdf).
} 
pathological (e.g. Alzheimer's disease) or physiological (e.g. normal aging) development of brains. Therefore, an accurate cortical thickness measuring method with longitudinal consistency and stability, which can detect and monitor the developmental changes of cortical thickness, is highly desirable. Many cortical thickness methods have been previously proposed. They can be broadly categorized as explicit surface based, implicit surface based and probabilistic segmentation based. In the explicit surface-based methods, after the inner (WM/GM interface) and outer (GM/CSF interface) surfaces are extracted by deformable surface models (which incorporate the smoothness constraints), the thickness is defined as the distance between a pair of points from each of the two surfaces. The correspondence between the two points in the pair is found either by deformable mapping of the inner surface to the outer surface [1,2], nearest point [3] or surface normal 44. The disadvantages of using explicit surface are the extra computational cost and errors generated by the surface construction. In contrast, in implicit surface-based approaches, after segmentation of brain tissues, no surface mesh is explicitly constructed to represent the WM/GM and GM/CSF boundary. This makes the algorithms in this category more computationally efficient. The PDE-based [5] method is one of the representative approaches in this category [6, 7, 8. In this method, Laplace's equation is solved in the GM region with certain boundary conditions (by setting different constant potentials on the two boundaries). The nested sub-layers of cortex is thus revealed by the resultant iso-potentials. The cortical thickness is then defined at each point as the length of the streamline along the gradient of the defined potential field. Some of the above methods are based on a hard segmentation of brain tissues. The disadvantage of using hard segmentation is the losing of sub-voxel information, which makes the algorithms very sensitive to the segmentation errors. To overcome this limitation, methods which measure the thickness on probabilistic segmentation of GM are proposed. Diffeomorphic registration of the probabilistic segmentation image is used in [9] to find a one-to-one correspondence of point pairs, between which cortical thickness is defined. Similarly, in [10], thickness is defined as the minimum line integral across the probablistic GM segmentation.

The above existing methods can be considered as 3D thickness measuring approaches, because they are designed to measure the thickness temporalindependently and do not take into account the temporal correlation. In order to improve the measuring accuracy and stability in longitudinal thickness studies, in this paper, we aim to devise a $4 \mathrm{D}$ thickness measuring method which is capable of fully utilizing the temporal information provided by longitudinal image dataset. The reason why $4 \mathrm{D}$ thickness measurement is important and necessary is that, measuring cortical thickness from MR images is affected by many artifacts and noises, such as intensity inhomogeneity, partial volume (PV) effect and segmentation errors. Comparing the thickness of cortical structures $(1.2 \sim 4.5 \mathrm{~mm}[11])$ to the the resolution of MR images $(\approx 1 \mathrm{~mm})$, the errors introduced in the measuring process are considerably large. Therefore, if the thickness changes are evaluated as the difference between two temporal-independent 3D measurements, these errors will be amplified and result in jittery longitudinal 
measurements. Another fact makes the longitudinal study even more difficult is that the expected change in GM thickness during the early stages of some neurological disorders, e.g. Alzheimer's disease, has been shown to be less than $1 \mathrm{~mm}$ in most brain regions [12,13. Since the cortical structure are only a few voxels thick in the images, sub-voxel accuracy is required to detect the subtle longitudinal thickness changes. In this situation, incorporating the information from other time-points as constraints to improve the accuracy and robustness of thickness measurement becomes very important. Currently, such temporal constraints are introduced by applying some sort of regression over the independently estimated 3D measurements. The problem of this regression-based method is that it imposes overly restrictive constraints due to the limitation of the pre-assumed regression model (usually linear), without taking into account the temporal correlation. This prompts us to incorporate the temporal constraints directly into the thickness measurement process. In this paper, after getting the $4 \mathrm{D}$ segmentation results of the longitudinal input images using a $4 \mathrm{D}$ segmentation method [14, we propose to measure the thickness on the aligned GM probability maps of different time-points in a common stereotaxic space. In this way, information from all time-points can be easily incorporated.

\section{Methods}

\subsection{Cortical Thickness Measurement by Minimum Line Integral}

In [10], a 3D thickness measurement is defined as the minimum line integral on the probabilistic segmentation of GM. As shown in Fig. 1, the thickness on each voxel in the GM (denoted by red dot) is defined as the minimum line integral (denoted as yellow arrow) of the probability map of the GM (the underneath image) over all possible directions (denoted as a group of green arrows) passing through that voxel. Mathematically, this method can be expressed as:

$$
T(\mathbf{x})=\min _{l \in L_{\mathbf{x}}} \int_{l} P(\mathbf{x}) \mathrm{d} l
$$

where $T(\mathbf{x})$ is the measured thickness of cortex at voxel $\mathbf{x} \in \mathbb{R}^{3} . P(\mathbf{x}) \in\left[\begin{array}{ll}0 & 1\end{array}\right]$ is the probability of the point $\mathbf{x}$ belonging to the GM. $L_{\mathbf{x}}$ is the set of all possible lines in three-dimensional space passing through $\mathbf{x}$. In implementation, $L_{\mathbf{x}}$ is defined by evenly sampling on the unit sphere.

\subsection{D Cortical Thickness Measurement}

As Fig. 2 shows, our 4D thickness measurement method consists of five steps. Without loss of generality, we use a longitudinal image dataset with one baseline scan and $N-1$ follow-up scans to explain each step in the pipeline. 


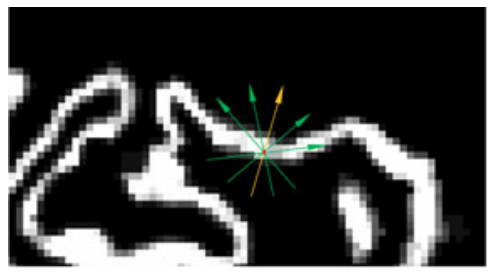

Fig. 1. Cortical thickness measurement by minimal line integral

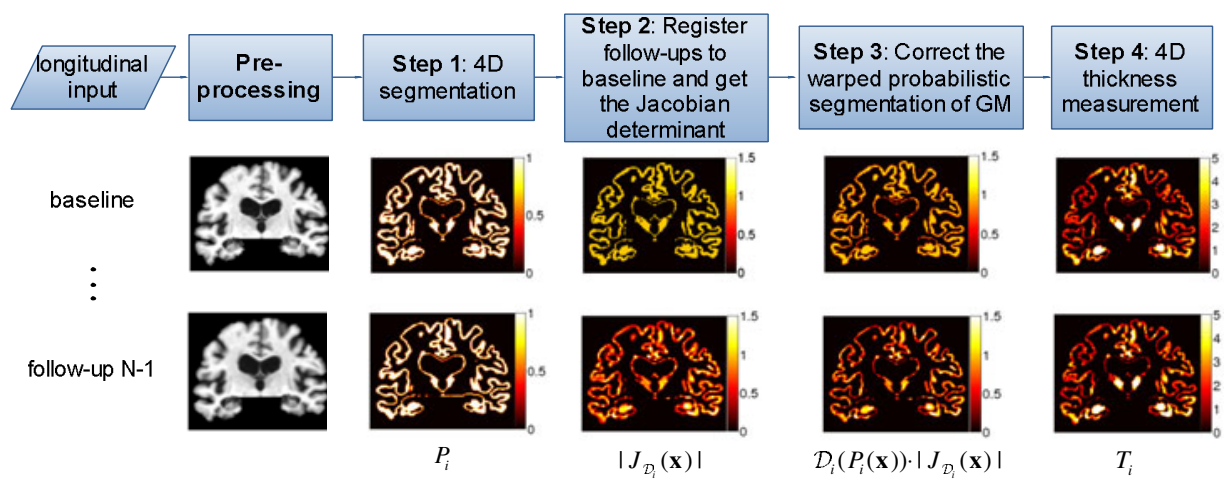

Fig. 2. 4D cortical thickness measurement

Pre-processing: Intensity inhomogeneity is firstly corrected [15]. Next, the $N-$ 1 follow-up images are rigidly (6 DOFs) registered to the baseline images, because the images are from the same subject with only translational or rotational misalignments. After that, the skull and cerebellum are removed [16] in baseline. Finally, by applying the resultant mask in baseline onto the aligned follow-up scans, we get consistent skull and cerebellum removing results in all followup scans.

Step 1: Input the pre-processed images into a 4D segmentation algorithm (CLASSIC [14]), to acquire the segmentation of GM with higher accuracy and longitudinal consistency. The probabilistic 4D segmentations of GM are denoted as $P_{i}, i=1, \cdots, N$.

Step 2: Register each follow-up to baseline using diffeomorphic demons registration [17. The resultant deformation fields and warped probabilistic segmentation of GM are denoted as $\mathcal{D}_{i}$ and $\mathcal{D}_{i}\left(P_{i}\right)$, respectively. To quantify the expansion and contraction caused by transformation $\mathcal{D}_{i}$, on each voxel, the Jacobian determinant $\left|J_{\mathcal{D}_{i}}(\mathbf{x})\right|$ are calculated (as shown in step 2 of Fig. 2). This map will be used as a scaling factor to correct the warped GM probability map $\mathcal{D}_{i}\left(P_{i}\right)$ by preserving the probabilistic volume. The reason to impose the 
diffeomorphic constraints in the inter-timepoint registration is to seek a minimal deforming path. This property makes the deformation along the radial direction (the direction thickness changes) on the cortex mantle. In [9, this property of diffeomorphic registration was also used to find the corresponding point pairs in thickness measurement.

Step 3: In order to preserve the probabilistic volume, the warped GM probability map $\mathcal{D}_{i}\left(P_{i}\right)$ is corrected by multiplying with the scaling factor:

$$
P_{i}^{\prime}(\mathbf{x})=\mathcal{D}_{i}\left(P_{i}(\mathbf{x})\right) \cdot\left|J_{\mathcal{D}_{i}}(\mathbf{x})\right|
$$

This local probabilistic volume preserving step is also known as modulation in voxel-based morphometry [18.

Step 4: In the order of the scan time, the warped and corrected GM probability map of each time-point can be represented as $P_{1}^{\prime}, \cdots, P_{N}^{\prime}$. Since these maps are in a standardized stereotaxic space (baseline space) and the probabilistic volume is preserved, for each GM voxel in this space, the minimal line integral direction can be defined as:

$$
l_{\text {min }}=\arg \min _{l \in L_{\mathbf{x}}} \frac{1}{N} \sum_{i=1}^{N} \int_{l} P_{i}^{\prime}(\mathbf{x}) \mathrm{d} l
$$

Different from the definition in 3D temporal-independent thickness measurement, $l_{\min }$ is the optimal thickness measuring direction not only for a single time-point, but for all the images in the longitudinal image dataset. This means information from different time-points is fully utilized as constraint and guidance in finding the optimal measuring direction, which is the key factor in thickness measuring algorithms. Therefore, $l_{\min }$ can be robustly estimated against the noises. The thickness at GM voxel $\mathbf{x}$ on the $i$-th time-point is then defined as the line integral on $P_{i}^{\prime}$ along $l_{\text {min }}$ :

$$
T_{i}(\mathbf{x})=\int_{l_{\text {min }}} P_{i}^{\prime}(\mathbf{x}) \mathrm{d} l
$$

In this $4 \mathrm{D}$ measurement, we can make sure that the thicknesses to be compared in the longitudinal study are based on a common measuring direction. This will make all the thickness values resistant to outliers and much more comparable than the 3D measurement.

\section{Experiments and Results}

The validation of cortical thickness measuring algorithm has been a difficult problem, because no gold standard is available and can be used to evaluate a measurement. It is very difficult to manually measure the thickness in 3D images, due to the highly convoluted nature of cortex. Instead of the direct 
validation, indirect validation method has been adopted to compare different thickness measuring methods [10,19. This method is based on the fact that cortical thickness has close relationship with the psychological functions of brain. In some diseases, such as Alzheimer's disease, the decrease of thickness has been found highly correlated to the psychological disorder [20,21] which can be quantified by scores from some clinical examination, such as Mini-MentalStatus-Examination (MMSE) or Clinical Dementia Rating (CDR). Therefore, by comparing the correlations detected by different measuring method and the corresponding statistical significance, the accuracy and reliability of each method when applied in clinical studies can be evaluated [19.

Data. Data used in the experiment were obtained from the public available ADNI database (www.loni.ucla.edu/ADNI). In our study, 40 patients with mild cognitive impairment (MCI) who later developed to probable Alzheimer's disease (AD) and, for comparison, 15 normal controls (NC) were selected. In MCI group, the average MMSE decline is 9.2 (within 2 3 years), which indicates a substantial neuropsychological disorder has been developed. Therefore, the

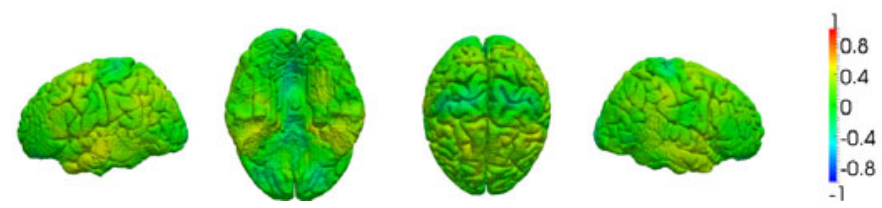

(a) $3 \mathrm{~S} 3 \mathrm{~T}$


(b) $3 \mathrm{~S} 4 \mathrm{~T}$
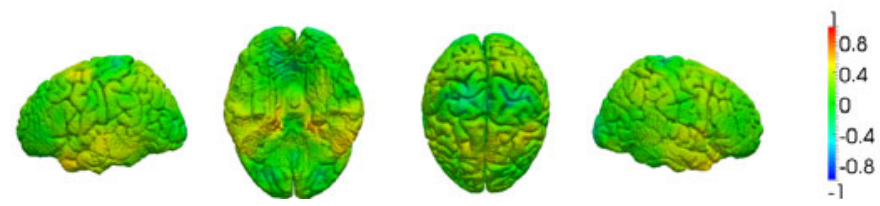

(c) $4 \mathrm{~S} 3 \mathrm{~T}$


(d) $4 \mathrm{~S} 4 \mathrm{~T}$

Fig. 3. Average correlation between thickness and MMSE scores. From left to right: the left, inferior, superior and right views. 
corresponding decrease of the cortical thickness is expected. In $\mathrm{NC}$ group, the average MMSE change is 1.3. Considering the possible MMSE assessment errors, subjects in this group can be regarded as neuropsychological healthy, and thus the cortical thickness is expected to keep stable (or slight decrease with normal aging).

Experiment design. Since the $4 \mathrm{D}$ processing is introduced at both the segmentation step and the thickness measuring step, totally four different thickness measuring pipelines are compared in order to trace the source of the possible observed improvements. The four different combinations are: 3D segmentation and 3D thickness measurement (3S3T), 3D segmentation and 4D thickness measurement (3S4T), 4D segmentation and 3D thickness measurement (4S3T) and $4 \mathrm{D}$ segmentation and $4 \mathrm{D}$ thickness measurement (4S4T). After the 4 different thickness values are measured, in order to conduct voxel-wise group analysis, each subject's thickness maps are mapped onto the template space.

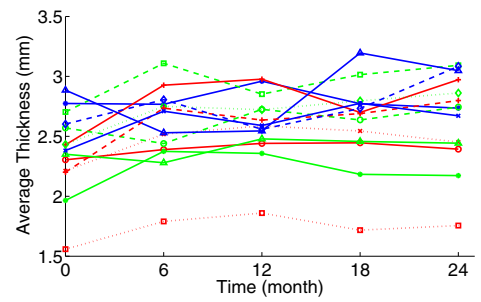

(a) $3 \mathrm{~S} 3 \mathrm{~T}$

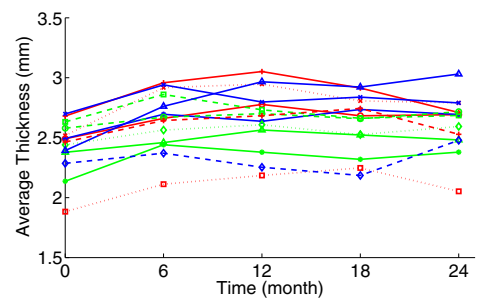

(c) $4 \mathrm{~S} 3 \mathrm{~T}$

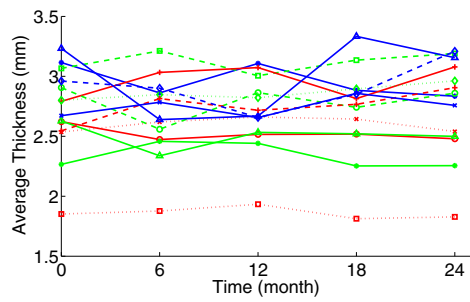

(b) $3 \mathrm{~S} 4 \mathrm{~T}$

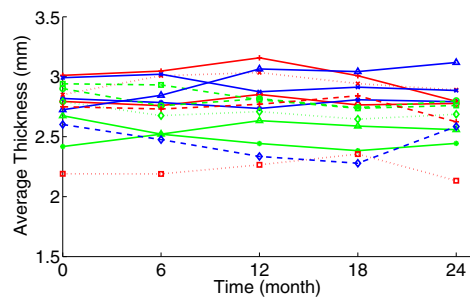

(d) $4 \mathrm{~S} 4 \mathrm{~T}$

Fig. 4. Longitudinal change of average thickness of 15 subjects from NC group. (Each curve represents a subject).

Results on MCI group. Before the voxel-wise correlation analysis can be conducted in the template space, the mapped thickness is first smoothed using full-width-at-half-maximum (FWHM) Gaussian filter $(\sigma=8 \mathrm{~mm})$ in order to suppress possible registration errors and inter-subject structure variations. After that, Pearson's correlation between thickness and MMSE score is calculated voxel-wise within each subject. The average correlation within the MCI 
Table 1. Longitudinal stabilities of different thickness measuring methods

\begin{tabular}{lcccc}
\hline \hline & $3 \mathrm{~S} 3 \mathrm{~T}$ & $3 \mathrm{~S} 4 \mathrm{~T}$ & $4 \mathrm{~S} 3 \mathrm{~T}$ & $4 \mathrm{~S} 4 \mathrm{~T}$ \\
\hline regression residual & & & & \\
$($ mean \pm std, $\mathrm{mm})$ & $0.161 \pm 0.067$ & $0.121 \pm 0.073$ & $0.117 \pm 0.052$ & $0.081 \pm 0.040$ \\
\hline
\end{tabular}

group are then computed by transforming the correlation coefficients to Fisher's $z$-value and transforming back. The resultant average correlations from the four methods are summarized in Fig. 3. As we can see, when more 4D components are added into the pipeline, the higher correlation can be detected. The average correlation detected increases in the order $3 S 3 T<3 S 4 T$ and $4 S 3 T<4 S 4 T$. Among all the four methods, the fully 4D method (4S4T) gives the highest correlation. This shows that both $4 \mathrm{D}$ segmentation and $4 \mathrm{D}$ thickness measurement can improve the accuracy and consistency for longitudinal thickness analysis. Superior/Mid Temporal Pole, Entorhinal Cortex and Middle/Inferior Temporal Gyrus are the five ROIs, in which the highest correlation are detected. These findings are consistent with those from [20,21.

Results on NC group. In the experiment on NC group, the stability (robustness) of different methods are compared in the situation that the change of thickness is very slight. For each subject, the average whole cortical thickness is computed at every time-point. This longitudinal average thickness change of the $15 \mathrm{NC}$ subjects are shown in Fig. 4. As we can see, 3D methods generate jittery changes which indicates the lack of longitudinal stability and consistency. In contrast, the proposed method is much more resistant to the noise and gives the most stable thickness measures. To quantitatively compare this stability, for each subject, a linear regression is performed on the longitudinal change curve and the fitting errors (residual) are computed. The results are summarized in Table 1, Our method gives the smallest mean residual (which can be viewed as the estimated mean error) and the smallest standard deviation.

\section{Conclusion}

We presented a 4D cortical thickness measuring framework. By applying the minimal line integral thickness measuring method on the aligned probability maps of GM from each time-point, we incorporate longitudinal information into the thickness measurement as temporal constraints. Experiments on clinical images from ADNI show that our method can detect much higher correlation between cortical thickness and MMSE scores with higher statistical significance. This indirectly indicates that our method is much more consistent and accurate in thickness measurement for longitudinal data. 


\section{References}

1. Fischl, B., Dale, A.M.: Measuring the thickness of the human cerebral cortex from magnetic resonance images. Proc. Natl. Acad. Sci. USA 97(20), 11050-11055 (2000)

2. Zeng, X., Staib, L., Schultz, R., Duncan, J.: Segmentation and measurement of the cortex from 3-D mr images using coupled-surfaces propagation. IEEE Trans. Med. Imag. 18(10), 927-937 (1999)

3. Miller, M., Massie, A., Ratnanather, J., Botteron, K., Csernansky, J.: Bayesian construction of geometrically based cortical thickness metrics. NeuroImage 12(6), 676-687 (2000)

4. Scott, M., Thacker, N.: Robust tissue boundary detection for cerebral cortical thickness estimation. In: Duncan, J.S., Gerig, G. (eds.) MICCAI 2005. LNCS, vol. 3750, pp. 878-885. Springer, Heidelberg (2005)

5. Jones, S., Buchbinder, B., Aharon, I.: Three-dimensional mapping of cortical thickness using laplace's equation. Hum. Brain Mapp. 11(1), 12-32 (2000)

6. Yezzi, A.J., Prince, J.L.: An Eulerian PDE approach for computing tissue thickness. IEEE Trans. Med. Imaging 22(10), 1332-1339 (2003)

7. Hutton, C., De Vita, E., Ashburner, J., Deichmann, R., Turner, R.: Voxel-based cortical thickness measurements in MRI. Neuroimage 40(4), 1701-1710 (2008)

8. Acosta, O., Bourgeat, P., Zuluaga, M.A., Fripp, J., Salvado, O., Ourselin, S.: Automated voxel-based 3D cortical thickness measurement in a combined LagrangianEulerian PDE approach using partial volume maps. Med. Imag. Analysis 13(5), 730-743 (2009)

9. Das, S.R., Avants, B.B., Grossman, M., Gee, J.C.: Registration based cortical thickness measurement. NeuroImage 45(3), 867-879 (2009)

10. Aganj, I., Sapiro, G., Parikshak, N., Madsen, S.K., Thompson, P.M.: Measurement of cortical thickness from MRI by minimum line integrals on soft-classified tissue. Hum. Brain Mapp. 30(10), 3188-3199 (2009)

11. von Economo, C.: The Cytoarchitectonics of the Human Cerebral Cortex. Oxford Medical Publications, London (1929)

12. Lerch, J., Pruessner, J., Zijdenbos, A., Hampel, H., Teipel, S., Evans, A.: Focal decline of cortical thickness in Alzheimers disease identified by computational neuroanatomy. Cerebral Cortex 15(7), 995-1001 (2005)

13. Singh, V., Chertkow, H., Lerch, J., Evans, A., Dorr, A., Kabani, N.: Spatial patterns of cortical thinning in mild cognitive impairment and Alzheimers disease. Brain 129(11), 2885-2893 (2006)

14. Xue, Z., Shen, D., Davatzikos, C.: CLASSIC: Consistent longitudinal alignment and segmentation for serial image computing. Neuroimage 30(2), 388-399 (2006)

15. Sled, J.G., Zijdenbos, A.P., Evans, A.C.: A nonparametric method for automatic correction of intensity nonuniformity in MRI data. IEEE Trans. Med. Imag. 17(1), 87-97 (1998)

16. Smith, S.: Fast robust automated brain extraction. Hum. Brain Mapp. 17(3), 143$155(2002)$

17. Vercauteren, T., Pennec, X., Perchant, A., Ayache, N.: Diffeomorphic demons: Efficient non-parametric image registration. NeuroImage 45(1S1), S61-S72 (2009)

18. Ashburner, J., Friston, K.J.: Voxel-based morphometry-the methods. NeuroImage $11(6), 805-821(2000)$ 
19. Lerch, J.P., Evans, A.C.: Cortical thickness analysis examined through power analysis and a population simulation. NeuroImage 24(1), 163-173 (2005)

20. Desikan, R.S., Cabral, H.J., Hess, C.P., Dillon, W.P., Glastonbury, C.M., Weiner, M.W., Schmansky, N.J., Greve, D.N., Salat, D.H., Buckner, R.L., Fischl, B.: Automated MRI measures identify individuals with mild cognitive impairment and Alzheimer's disease. Brain 132(8), 2048-2057 (2009)

21. Holland, D., Brewer, J.B., Hagler, D.J., Fenema-Notestine, C., Dale, A.M.: Subregional neuroanatomical change as a biomarker for Alzheimer's disease. Proceedings of the National Academy of Sciences 106(49), 20954-20959 (2009) 